ACCEPTED MANUSCRIPT

\title{
Synthesis and characterization of rGO-graft-Poly(Trimethylene Carbonate) for nerve regeneration conduits
}

To cite this article before publication: Zhengchao Guo et al 2019 Biomed. Mater. in press https://doi.org/10.1088/1748-605X/ab0269

\section{Manuscript version: Accepted Manuscript}

Accepted Manuscript is "the version of the article accepted for publication including all changes made as a result of the peer review process, and which may also include the addition to the article by IOP Publishing of a header, an article ID, a cover sheet and/or an 'Accepted Manuscript' watermark, but excluding any other editing, typesetting or other changes made by IOP Publishing and/or its licensors"

This Accepted Manuscript is @ 2018 IOP Publishing Ltd.

During the embargo period (the 12 month period from the publication of the Version of Record of this article), the Accepted Manuscript is fully protected by copyright and cannot be reused or reposted elsewhere.

As the Version of Record of this article is going to be / has been published on a subscription basis, this Accepted Manuscript is available for reuse under a CC BY-NC-ND 3.0 licence after the 12 month embargo period.

After the embargo period, everyone is permitted to use copy and redistribute this article for non-commercial purposes only, provided that they adhere to all the terms of the licence https://creativecommons.org/licences/by-nc-nd/3.0

Although reasonable endeavours have been taken to obtain all necessary permissions from third parties to include their copyrighted content within this article, their full citation and copyright line may not be present in this Accepted Manuscript version. Before using any content from this article, please refer to the Version of Record on IOPscience once published for full citation and copyright details, as permissions will likely be required. All third party content is fully copyright protected, unless specifically stated otherwise in the figure caption in the Version of Record.

View the article online for updates and enhancements. 


\title{
Synthesis and Characterization of rGO-graft-Poly(Trimethylene Carbonate) for Nerve Regeneration Conduits
}

\author{
Zhengchao Guo ${ }^{1}$, Sandra Kofink ${ }^{1}$, Honglin Chen ${ }^{1,3}$, Jia Liang ${ }^{1}$, Dirk W. Grijpma ${ }^{1,2}$, André A. Poot ${ }^{1 *}$ \\ Corresponding Author: a.a.poot@utwente.nl \\ ${ }^{1}$ Dept. of Biomaterials Science and Technology, University of Twente, Enschede, The Netherlands \\ ${ }^{2}$ Dept. of Biomedical Engineering, University Medical Center Groningen, Groningen, The Netherlands \\ ${ }^{3}$ Institute for Life Science, School of Medicine, South China University of Technology, China
}

\begin{abstract}
Graphene-graft-polymer has been used to improve the compatibility between graphene and a polymer matrix, and to further enhance electrical, mechanical and biological properties of polymer/graphene composites. In this study, poly(trimethylene carbonate) (PTMC) was successfully grafted onto graphene surface via "grafting from" method. Reduced graphene oxide (rGO) initiator was synthesized by azido ethanol reaction with graphene oxide (GO) at high temperature. This resulted in thermal reduction of the GO and stable hydroxyl groups on the graphene surface. Subsequently, rGO initiator was used for the ringopening polymerization of TMC monomer. rGO-graft-PTMC composites with PTMC molecular weights of 430, 480, 2150 and $7030 \mathrm{~g} / \mathrm{mol}$ were successfully synthesized using different amounts of TMC. Single layer graphene nanosheets remained after graft polymerization by this method. rGO-graft-PTMC dispersions in chloroform were stable. The rGO-graft-PTMC composites with PTMC molecular weights of 430-7030 $\mathrm{g} / \mathrm{mol}$ had electrical conductivities ranging from $0.2-0.016 \mathrm{~S} / \mathrm{cm}$. To investigate the biocompatibility of rGO-graft-PTMC, PTMC-based films containing rGO-graft-PTMC were prepared and used in cell culturing experiments. The composite films showed good biocompatibility with PC12 neuronal cells. It is concluded that rGO-graft-PTMC composite is a promising material for the preparation of nerve regeneration conduits.
\end{abstract}

Key words: Poly(trimethylene carbonate) (PTMC), Graphene, rGO-graft-PTMC, Graft polymerization, Electrical conductivity, Peripheral nerve regeneration.

\section{Introduction}

Peripheral nerve regeneration in the case of long-gap peripheral nerve damage remains a challenge [1, 2]. At the moment there are three methods for the treatment of peripheral nerve injury. End-to-end suturing is the first method in which the two ends of nerve stumps are sewed together using a biodegradable suture. This method fails if the gap is too long. Then an alternative procedure, autologous nerve grafting can be applied. This is referred as the "gold standard" [3]. However, it comes with disadvantages as the number of donor nerves is limited and additional surgery is needed [4]. Hence, tubular nerve guide conduits have been developed to connect both nerve ends as a bridging device, providing a suitable environment for the regenerating herve without scar tissue and compression from other tissues $[5,6]$.

To prepare such a nerve guide conduit, a material with controlled biodegradability and biocompatibility is needed. Poly(ethylene glycol) (PEG) [7], poly(e-caprolactone) (PCL) [8], poly(lactic acid) (PLA) [9] and 
poly(lactic-co-glycolic acid) (PLGA) [10] are well known polymers and have been used for the preparation of nerve guide conduits. Although promising results were obtained, these materials have drawbacks in terms of mechanical properties, degradation behavior and release of acidic degradation products, warranting the use of other polymers. Since nerve guide conduits are implanted by surgery it is required that the material can handle stress that occurs due to handling and suturing [11]. Additional properties are a low degree of swelling to reduce pressure on the nerve. Flexibility, permeability [12] and toughness [13] are required as well. Recently, poly(trimethylene carbonate) (PTMC) has attracted a lot of attention due to its excellent biocompatibility, surface degradation behavior and adjustable mechanical properties. Rubber-like PTMC can be synthesized by ring-opening polymerization of 1,3-trimethylene carbonate (TMC) and is already used for different biomedical applications [14]. For the fabrication of polymer structures such as nerve guide conduits, especially creep- and tear-resistant PTMC networks are interesting [15].

Stimulation of neuronal cell growth and differentiation has been observed on electrically conductive interfaces. This initiated the use of electrically conductive materials in research on nerve regeneration [16]. Graphene in the form of graphene oxide (GO) and reduced graphene oxide (rGO) shows promising physical and chemical properties to promote neuronal cell growth $[17,18]$. Hence, graphene-based composites were used to fabricate nerve guide conduits which showed strong potential to restore nerve function [19-21]. In our previous study [22], rGO/PTMC composites showed electrical conductivity and facilitated the growth of neuronal cells. While preparing the graphene/polymer composites, however, irreversible aggregates of graphene layers formed due to van der Waals interactions [23]. This can be prevented by using functionalized graphene nanosheets in the form of graphene-graft-polymer [24]. Dispersion of graphenegraft-polymer in a polymer solution improves not only the processing ability but also the thermal stability and mechanical properties of the prepared structures [25-27]. "Graft to" and "Graft from” techniques have been used to synthesize graphene-graft-polymers. Generally, "Graft from” results in a higher graft density and is more likely to yield thin graphene sheets [28]. Graphene sheets with thermally stable hydroxyl groups, designated as rGO initiator, can be prepared by nitrene chemistry as reported by Gao [29]. In the present work, rGO-graft-PTMC composites with a varying PTMC content were synthesized by the ring-opening polymerization of TMC with rGO initiator. After physical and chemical characterization, including determination of electrical conductivity, the biocompatibility of PTMC-based composite films was investigated by culturing experiments with PC12 neuronal cells.

\section{Materials and methods}

\subsection{Materials}

Sulfuric acid ( $\left.\mathrm{H}_{2} \mathrm{SO}_{4}, 98.0 \%\right)$, phosphorus pentoxide (99 \%), ammonium persulfate (98.0 \%), potassium permanganate $(99.0 \%)$, hydrogen peroxide solution $\left(\mathrm{H}_{2} \mathrm{O}_{2}, 30 \%\right.$ in water), hydrochloric acid solution (HCL, $37 \%$ in water), 2-chloroethanol (99\%), sodium azide (99.5\%), stannous octoate (Sn(Oct) 2 ), 1- 4(2-Hydroxyethoxy)-phenyl -2-hydroxy-2-methyl-1-propane-1-one (Irgacure 2959) and magnesium sulfate $\left(\mathrm{MgSO}_{4}\right)$ were purchased from Sigma Aldrich, The Netherlands. $\mathrm{MgSO}_{4}$ was dried in an oven at $160^{\circ} \mathrm{C}$ before use, the other chemicals were used as delivered. Graphite flake (natural, -325 mesh, 99.8 \%) was acquired from Alfa Aesar, Germany. Diethyl ether, acetone, toluene and dichloromethane (DCM) were bought from VWR Chemicals, Germany. N-methyl-pyrrolidone (NMP) was purchased from Merck, Germany. TMC Monomer was kindly provided by Huizhou Foryou Medical Devices, China. PC12 cells (ATCC $^{\circledR}$ CRL-1721 ${ }^{\mathrm{TM}}$ ) were ordered from ATCC, Germany. RPMI-1640 culture medium and horse serum were purchased from Sigma Aldrich. Fetal bovine serum and penicillin/streptomycin were obtained from Gibco. PrestoBlue ${ }^{\mathrm{TM}}$ Cell Viability Reagent was ordered from Thermo Fisher Scientific. 


\subsection{Preparation of rGO initiator}

GO was synthesized by modified Hummers' method [30], as described in detail in our previous study [22]. A nitrogen chemistry reaction was performed to obtain thermally stable hydroxyl groups on the surface of the graphene layers. Homemade azido ethanol was used, synthesized according to [31]. Briefly, a $1000 \mathrm{~mL}$ three-neck-round-bottom flask was fitted with a condenser. Sodium azide ( $97.5 \mathrm{~g} ; 1.5 \mathrm{~mol}$ ), deionized water $(390 \mathrm{~mL})$ and 2-chloroethanol $(60.4 \mathrm{~g} ; 0.75 \mathrm{~mol})$ were added. The flask was heated to $75^{\circ} \mathrm{C}$ in an oil bath and kept stirring for 96 hours. After cooling to room temperature, the product was extracted with diethyl ether $\left(5\right.$ x $50 \mathrm{~mL}$ ). The extract was dried over anhydrous $\mathrm{MgSO}_{4}$ overnight. After filtering and evaporation of diethyl ether, azido ethanol was obtained as a colorless oil.

Commonly, ring-opening polymerization of TMC is performed at high temperature in the bulk [32, 33]. Thus, for the synthesis of rGO-graft-PTMC, thermally stable hydroxyl groups are needed to initiate the ringopening polymerization of TMC. Therefore, GO was not used as initiator but rGO initiator was synthesized according to the method by Gao et al. [34] as shown in Scheme 1. In detail, $500 \mathrm{mg}$ GO and $200 \mathrm{~mL}$ NMP were put into a bottle. It was sonicated for 120 minutes $(40 \mathrm{kHz}$, pre-experiments showed no formation of aggregates for this duration) and then placed in a $500 \mathrm{~mL}$ flask in an oil bath. After bubbling with nitrogen for 30 minutes, homemade azido ethanol (10 g) was added. The mixture, was heated to $160{ }^{\circ} \mathrm{C}$ and kept at this temperature for 18 hours in argon atmosphere under/constant știrring. During this step, GO was thermally reduced to rGO while reacting with azido ethanol to becomé rGO initiator. After cooling to room temperature, rGO initiator was separated by filtering the dispersion and washing with acetone. Finally, it was dried in a vacuum oven at room temperature until a constant weight was gained.

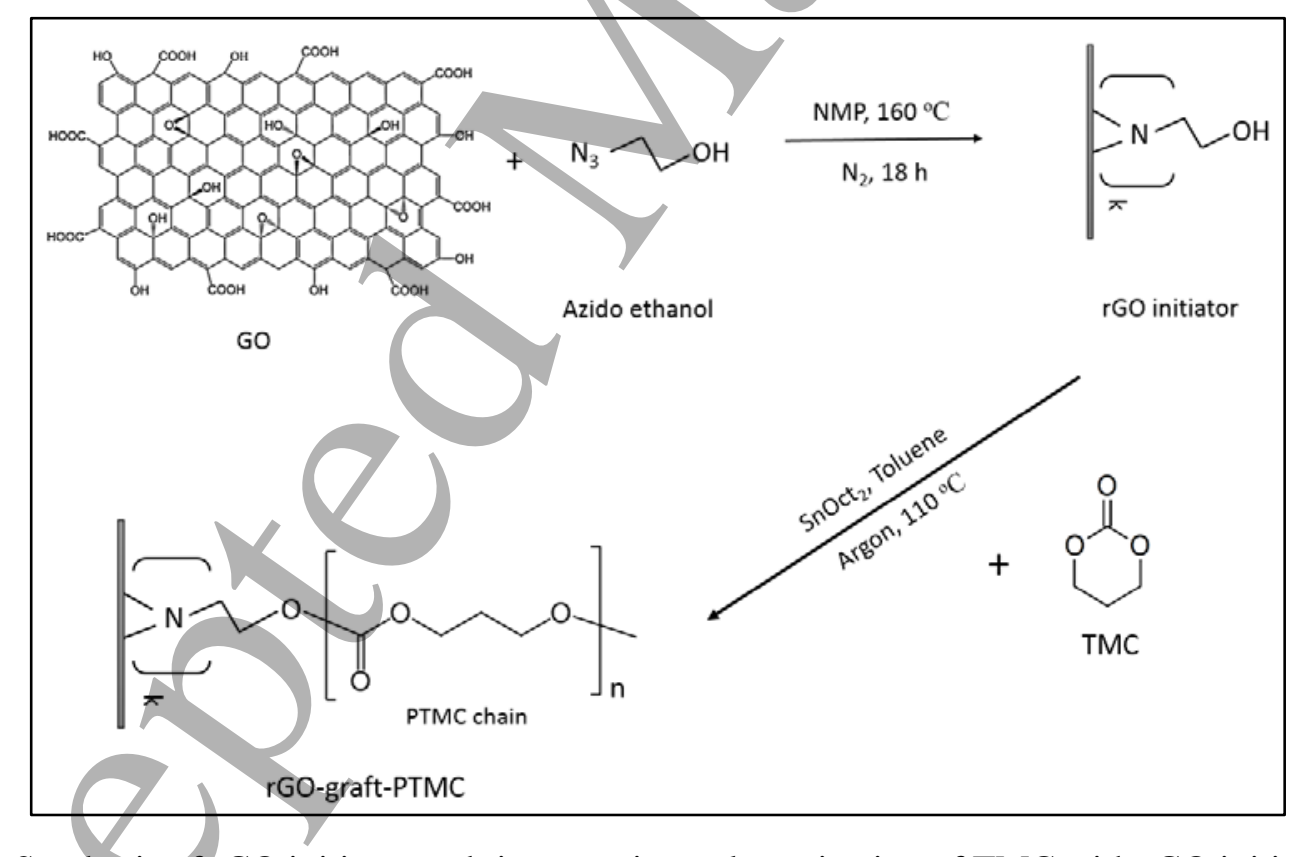

Scheme 1. Synthesis of rGO initiator and ring-opening polymerization of TMC with rGO initiator.

\subsection{Ring-opening polymerization of TMC with rGO initiator in toluene}

Toluene was chosen to disperse rGO initiator and dissolve TMC for the ring-opening polymerization of TMC. Different amounts of TMC monomer (2.5, 5, 10 and 15 g), 0.05 g rGO initiator and $15 \mathrm{~mL}$ toluene 
were added into three-neck flasks and sonicated for 40 minutes. The dispersions were heated up to $80{ }^{\circ} \mathrm{C}$ in an oil bath and $\mathrm{Sn}(\mathrm{Oct})_{2}(0.02 \mathrm{~mol} / \mathrm{g}$ monomer) was added as catalyst. The temperature was increased to $110{ }^{\circ} \mathrm{C}$ and left to react for 4 days under argon atmosphere with vigorous stirring, see Scheme 1 . After reaction, the flasks were cooled to room temperature and toluene was poured out. To remove homo PTMC and residual TMC monomer, the mixture was washed with DCM and rGO-graft-PTMC was separated by centrifugation at $5000 \mathrm{rpm}$ for 10 minutes. This washing/centrifugation step was performed 5 times. Púre rGO-graft-PTMC was obtained by drying in the vacuum oven at room temperature until all DCM was removed.

\subsection{Characterization}

Fourier-transform infrared spectroscopy (FTIR, PerkinElmer Spectrum Two) was used to characterize the chemical structure of GO, rGO initiator and rGO-graft-PTMC.

Thermogravimetric analysis (TGA, PerkinElmer Pyris 1) of GO, rGO initiator and rGO-graft-PTMC was performed with a temperature range from $50{ }^{\circ} \mathrm{C}$ to $550{ }^{\circ} \mathrm{C}$ and heating rate of $20^{\circ} \mathrm{C} / \mathrm{min}$ under a nitrogen flow $(20 \mathrm{~mL} / \mathrm{min})$.

X-ray diffractometry (XRD) was conducted on a PANalytical X'Pert Pro with Cu-K $\alpha$ radiation $(\lambda=0.15405$ $\mathrm{nm})$ at $45 \mathrm{kV}$ and $40 \mathrm{~mA}$.

X-ray photoelectron spectroscopy (XPS, Physical Electronics Quantera SXM) was performed using an Al $\mathrm{K} \alpha \mathrm{X}$-ray source $(1486.6 \mathrm{eV})$ and a vacuum pressure of $2.10^{-8}$ Torr. Multipack v.9.8 software was used for data analysis. For all these characterizations, dried powders of GO, rGO initiator and rGO-graft-PTMC were used.

\subsection{Morphology}

High resolution scanning electron microscopy (SEM, Zeiss MERLIN HR-SEM) and transmission electron microscopy (TEM, Philips CM300ST-FEG) were applied to observe the morphology of rGO initiator and rGO-graft-PTMC. To prepare the samples, diluted rGO initiator and rGO-graft-PTMC dispersions (around $0.05 \mathrm{mg} / \mathrm{mL}$ ) were prepared in chloroform and dropped on sample holders.

\subsection{Electrical Conductivity}

Electrical conductivity measurements were carried out by van der Pauw method [35, 36]. To prepare the samples, rGO initiator and rGO-graft-PTMC were firstly dispersed in chloroform with 40 minutes sonication below $30^{\circ} \mathrm{C}$. Then the dispersions were filtered using $0.2 \mu \mathrm{m}$ pore size filter paper to get thin films, which were dried in the air and cut into $5 \mathrm{~mm}$ width and $5 \mathrm{~mm}$ length specimens with a graphene layer thickness of around $0.1 \mathrm{~mm}$.

\subsection{Biocompatibility}

2.7.1 Preparation of PTMC-based composite films as substrates for cell culturing

PTMC-based films containing either rGO-graft-PTMC or rGO initiator were prepared as follows. Photocrosslinkable three-armed PTMC macromer with a molecular weight of 17,000 g/mol, functionalized with methacrylate end groups (PTMC-MA) was synthesized as previously reported [15]. Suspensions of rGOgraft-PTMC (sample code 2.5, see Table 1) and rGO initiator in chloroform were sonicated for 2 hours. Subsequently, PTMC-MA and photo initiator Irgacure 2959 (2.5 wt\% relative to PTMC-MA) were added to the dispersions. In both cases, the amount of rGO was $1 \mathrm{wt} \%$ relative to PTMC-MA. The mixtures were blended for 30 minutes and casted on glass plates to form thin films. The composite films were dried in the dark for 1 day at room temperature and then irradiated with $365 \mathrm{~nm}$ UV light for 30 minutes. Also plain 


\section{RESULTS AND DISCUSSION}

\subsection{Synthesis and characterization of rGO-graft-PTMC}

rGO-graft-PTMC was synthesized by ring-opening polymerization of TMC with rGO initiator in toluene. rGO initiator was used because of its thermal stability. Although there are large numbers of hydroxyl and carboxylic acid groups on the surface of GO, these groups are not thermally stable. Thermogravimetric analysis of GO, rGO initiator and different types of rGO-graft-PTMC was performed, as shown in Figure 1. $\mathrm{GO}$ was not thermally stable as mass loss started below $110^{\circ} \mathrm{C}$ due to loss of oxygen-containing groups like $-\mathrm{OH}$ and -COOH. Similar results were reported by Stankovich et al. [36]. During synthesis of rGO initiator at $160{ }^{\circ} \mathrm{C}$, these unstable groups were removed, GO was thermally reduced and at the same time azido ethanol was bound to the surface yielding rGO initiator. As shown in Figure 1, there was no significant mass loss of rGO initiator below $110{ }^{\circ} \mathrm{C}$, indicating that it was suitable to be used for the ring-opening polymerization of TMC at $110^{\circ} \mathrm{C}$. Grafted PTMC chains were degraded from $150{ }^{\circ} \mathrm{C}$ to $340{ }^{\circ} \mathrm{C}$. The degradation temperature of rGO-graft-PTMC increased with increasing PTMC content. It was also found that the grafted PTMC chains increased the thermal stability of graphene layers. All rGO-graft-PTMC samples started to degrade at a higher temperature than rGO initiator. Similar results were found by Fang et al. [23]. It was assumed that the weight loss of all rGO-graft-PTMC samples was completely due to decomposition of the PTMC chains. The initiator group content of rGO initiator as well as the PTMC content of the rGO-graft-PTMC composites could be estimated from the TGA measurements and were used to calculate the molecular weights of the grafted PTMC chains, as shown in Table 1. 
Table 1. Synthesis and analysis of rGO-graft-PTMC

Figure 1. TGA curves of GO, rGO initiator and rGO-graft-PTMC. 2.5, 5, 10 and 15 refer to the amount of TMC (g) used for polymerization.

\begin{tabular}{lccccc}
\hline Sample code & $\begin{array}{l}\text { rGO } \\
\text { initiator, }\end{array}$ & $\begin{array}{c}\text { TMC } \\
\text { mass, }\end{array}$ & $\begin{array}{c}\text { rGO-graft- } \\
\text { PTMC, }\end{array}$ & $\begin{array}{c}\text { Residual content } \\
\text { at } \mathbf{5 0 0}{ }^{\circ} \mathbf{C}, \mathbf{\%}\end{array}$ & $\begin{array}{c}\mathbf{M}_{\text {PTMC }} \text { by } \\
\text { TGA, g/mol }\end{array}$ \\
\hline rGO-graft-PTMC $\mathbf{2 . 5}$ & 0.05 & 2.5 & 0.0729 & 54.5 & 430 \\
\hline rGO-graft-PTMC 5 & 0.05 & 5 & 0.0680 & 51.6 & 480 \\
\hline rGO-graft-PTMC 10 & 0.05 & 10 & 0.1407 & 19.3 & 2150 \\
\hline rGO-graft-PTMC 15 & 0.05 & 15 & 0.6396 & 6.8 & 7030 \\
\hline
\end{tabular}

Initiator group content was $13.3 \mathrm{wt} \%$, which was the weight loss of rGO initiator in the temperature interval between $240{ }^{\circ} \mathrm{C}$ and $420^{\circ} \mathrm{C}$ [37]. M Initiator groups was $\mathrm{N}-\mathrm{CH}_{2}-\mathrm{CH}_{2}-\mathrm{OH}$ 's molar mass which is $59 \mathrm{~g} / \mathrm{mol}$. Average molecular weight of grafted PTMC chains (MPTMC) was calculated as follows. Mass $500{ }^{\circ} \mathrm{C}$ was the residual carbon

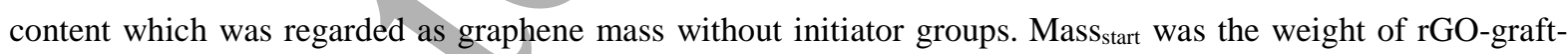
PTMC used for the TGA measurement.

$\mathrm{n}_{\mathrm{PTMC}}(\mathrm{mol})=\mathrm{n}_{\text {Initiator groups }}(\mathrm{mol})=\frac{\text { Mass }_{\text {Initiator groups }}}{\mathrm{M}_{\text {Initiator groups }}}=\frac{\operatorname{Mass}_{500}{ }^{\circ} \mathrm{C} * \frac{13.3 \%}{(100 \%-13.3 \%)}}{\mathrm{M}_{\text {Initiator groups }}}$.

Mass $_{500}{ }^{\circ} \mathrm{C}=$ Mass $_{\text {start }} *$ Residual content at $500{ }^{\circ} \mathrm{C}(\%)$.

$\mathrm{M}_{\text {PTMC }}=\frac{\text { Mass }_{\text {start }}-\text { Mass }_{500}{ }^{\circ} \mathrm{C}}{\mathrm{n}_{\text {PTMC }}}(\mathrm{g} / \mathrm{mol})$. 
Four kinds of rGO-graft-PTMC with different PTMC molecular weights were synthesized as shown in Table 1. With more monomer loading, the PTMC content and the molecular weight of grafted PTMC chains increased. In addition, both parameters dramatically increased at a monomer feeding over $10 \mathrm{~g}$, which can be explained by a more efficient polymerization at a higher monomer concentration. The average molecular weights of PTMC chains grafted on the rGO surfaces were calculated using the TGA data, see Table 1 and Figure 1. The PTMC molecular weights of the 4 different rGO-graft-PTMC composites were 430, 480, 2150 and $7030 \mathrm{~g} / \mathrm{mol}$, respectively.

FTIR spectroscopy was used to chemically characterize the synthesized GO, rGO initiator and rGO-graftPTMC, as shown in Figure 2A. Typical GO curves showed a broad band from 2900 to $3700 \mathrm{~cm}^{-1}$, which was the stretching peak of different hydroxyl groups. The peaks at 1716, 1635, 1152 and $1030 \mathrm{~cm}^{-1}$ were due to stretching of $\mathrm{C}=\mathrm{O}, \mathrm{C}=\mathrm{C}, \mathrm{C}-\mathrm{OH}$ and $\mathrm{C}-\mathrm{O}$ bonds, respectively. $\mathrm{rGO}$ initiator curves showed that unstable hydroxyl groups (2900-3700 $\mathrm{cm}^{-1}$ ) of GO were removed during azido ethanol reaction at high temperature. The peak around $1586 \mathrm{~cm}^{-1}$ belonged to $\mathrm{C}=\mathrm{C}$ and the one at $1164 \mathrm{~cm}^{-1}$ referred to remaining C-OH groups. Similar peaks were reported for thermally reduced graphene [38]. The FTIR spectrum of rGO-graft-PTMC showed C-O $\left(1230 \mathrm{~cm}^{-1}\right), \mathrm{C}=\mathrm{O}\left(1700 \mathrm{~cm}^{-1}\right)$ and $\mathrm{C}-\mathrm{H}\left(2900 \mathrm{~cm}^{-1}\right)$ peaks referring to PTMC chains grafted on the surface of rGO initiator.

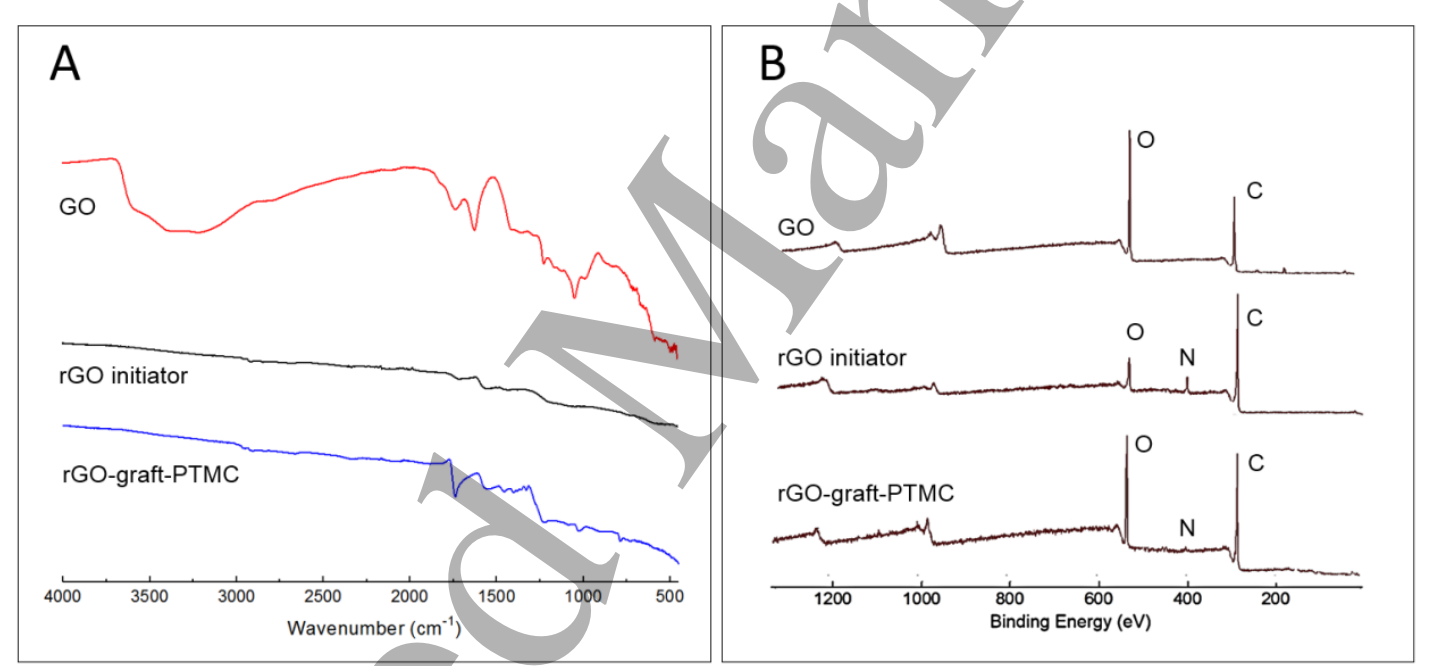

Figure 2. FTIR spectra (A) and XPS spectra (B) of GO, rGO initiator and rGO-graft-PTMC 2.5.

XPS spectra of GO, rGO initiator and rGO-graft-PTMC are shown in Figure 2B. In contrast to GO, rGO initiator showed a strong nitrogen signal, indicating that azido ethanol was coupled to the graphene surface. As compared to rGO initiator, rGO-graft-PTMC showed a stronger oxygen signal due to the presence of PTMC. The C, $\mathrm{N}$ and $\mathrm{O}$ atomic percentages of GO, rGO initiator and rGO-graft-PTMC are given in Table 2. As compared to GO, a significant nitrogen content was present in the case of rGO initiator. Thermal reduction of GO during synthesis of rGO initiator resulted in a strong decrease of oxygen content from 38.78 to $11.89 \%$, which increased to $20.80 \%$ upon graft polymerization of PTMC. The atomic percentages shown in Table 2 for rGO-graft-PTMC were determined after 5 times washing of the composite with DCM, in order to remove any adsorbed PTMC from the graphene surface. Additional 5 times washing of the rGOgraft-PTMC with DCM did not change the $\mathrm{C}, \mathrm{N}$ and $\mathrm{O}$ atomic percentages (data not shown). Taken together, this demonstrates that PTMC was successfully grafted onto the rGO surface.

Table 2. Atomic content of GO, rGO initiator and rGO-graft-PTMC as determined by XPS (n=4). 
XRD was used to verify exfoliation of graphene nanosheets in GO, rGO initiator and rGO-graft-PTMC. As shown in Figure 3, the GO pattern showed a strong peak around $10.1^{\circ}$, indicating the presence of oxygencontaining structures formed during oxidation. This strong peak disappeared in the patterns of rGO initiator and rGO-graft-PTMC, which is in agreement with thermal reduction during the synthesis of rGO initiator. The rGO initiator pattern showed a broad peak around $24.9^{\circ}$, similar to the pattern for thermally reduced graphene oxide reported by Mishra et al. [39]. The absence of a strong peak in the rGO initiator pattern indicates that the GO nanosheets remained exfoliated during the nitrene chemistry reaction [23]. The rGOgraft-PTMC pattern showed almost no visible peaks, suggesting stabilization of exfoliated rGO nanosheets during graft polymerization. Similar results were reported by Fang et al. [40].

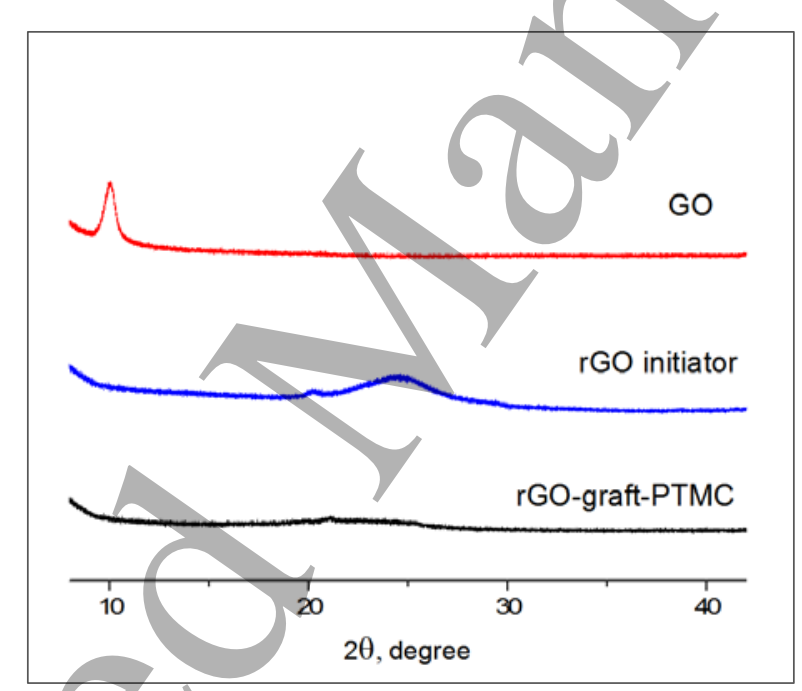

Figure 3. XRD curves of GO, rGO initiator and rGO-graft-PTMC 2.5. 


\subsection{Dispersion behavior}

The dispersion behavior of GO, rGO initiator and rGO-graft-PTMC was investigated in water and chloroform, see Figure 4 . The same concentration $(0.5 \mathrm{mg} / \mathrm{mL})$ was used for all samples. GO could be dispersed very well in water due to the large amount of hydroxyl groups resulting in its hydrophilic character. rGO initiator was not able to form a stable dispersion in water due to its relatively high hydrophobicity [41]. rGO-graft-PTMC could not be dispersed at all in water due to the hydrophobic PTMC chains on the graphene surface. In contrast to GO, rGO initiator and rGO-graft-PTMC could be stably dispersed in chloroform, which is a nonpolar solvent. To prepare a polymer/graphene composite, rGO initiator or rGO can easily form aggregates when it is mixed with polymer at a relatively high concentration. Grafted polymer chains on the surface of graphene increase the compatibility of the graphene with the polymer [42]. In the present study, grafting of PTMC chains on the surface of rGO nanosheets was applied to prepare homogenous PTMC/rGO-graft-PTMC composites using chloroform as a solvent.

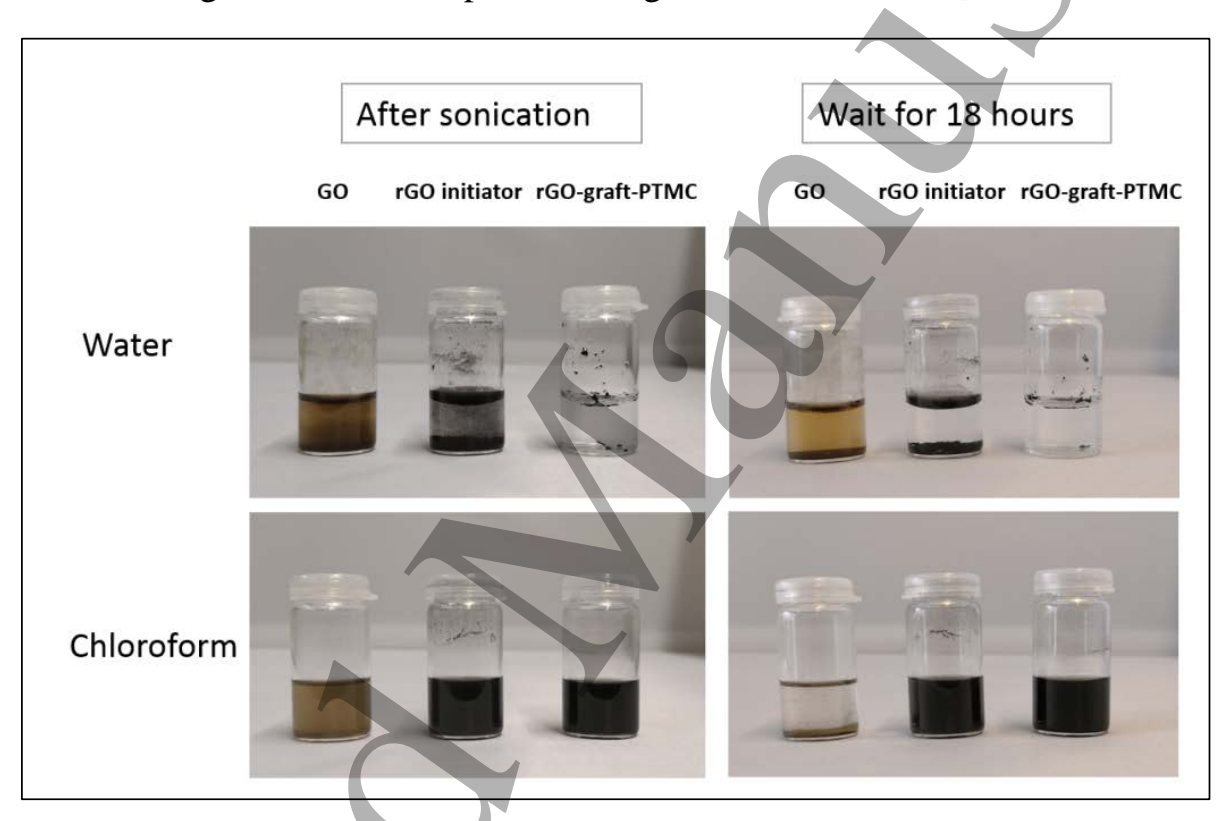

Figure 4. Dispersion behavior of GO, rGO initiator and rGO-graft-PTMC 2.5 in water and chloroform.

\subsection{Morphology}

The morphology of rGO initiator and rGO-graft-PTMC was investigated using both SEM and TEM. As shown in Figure 5A, the surface of rGO initiator was relatively smooth and clean. The surface of rGO-graftPTMC was rough due to the presence of a lot of PTMC particles (Figure 5B). This again proved that PTMC chains were successfully grafted on the surface of the rGO nanosheets. As shown in Figure 5C and D, single nanosheets of rGO initiator and rGO-graft-PTMC were present. Thus, single layer rGO nanosheets remained during the synthesis of graphene-graft-polymer. 

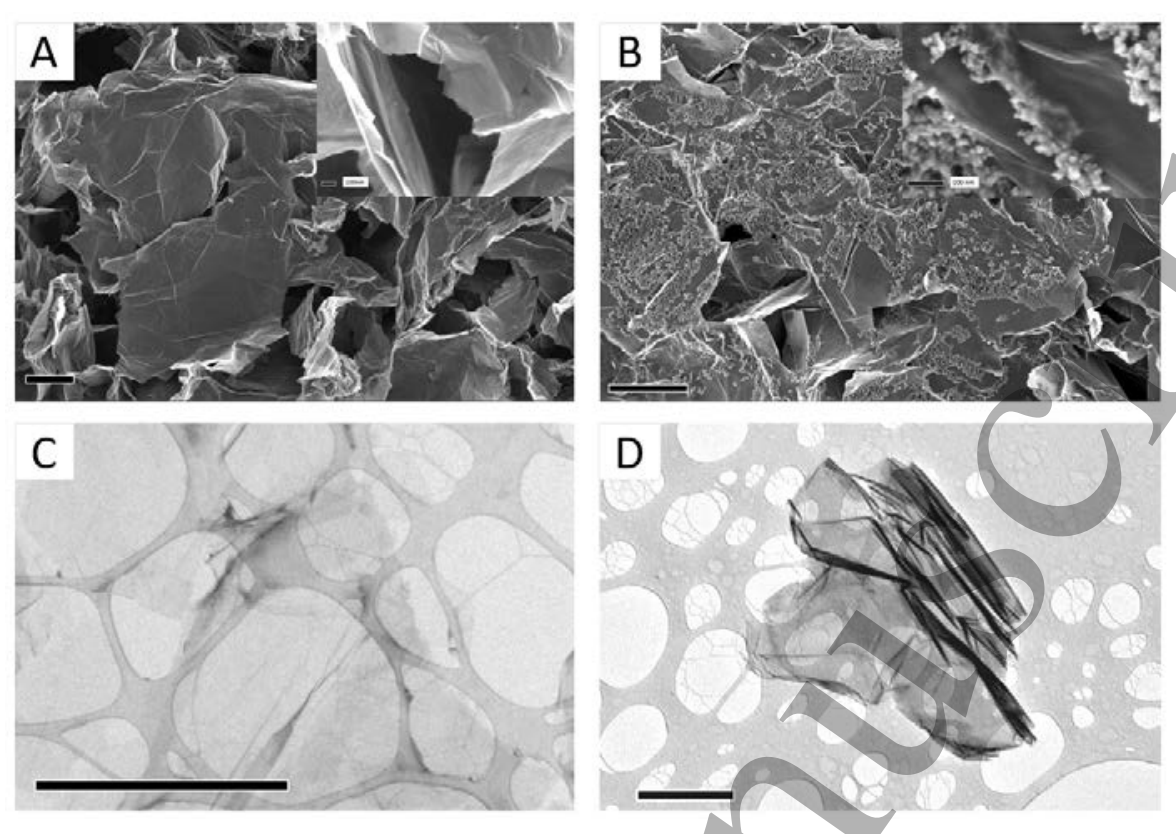

Figure 5. SEM images of rGO initiator (A) and rGO-graft-PTMC 2.5 (B), TEM images of rGO initiator (C) and rGO-graft-PTMC 2.5 (D), scale bar is $2 \mu \mathrm{m}$.

\subsection{Conductivity}

Electrical conductivity as the most important property of graphene derivatives was characterized as well, see Table 3. The electrical conductivity of graphene-based materials is related to their carbon content. In our case, a higher residual content at $500{ }^{\circ} \mathrm{C}$ indicates a higher amount of rGO in the rGO-graft-PTMC composites. As rGO initiator was thermally reduced, it showed a relatively high conductivity of $1.59 \mathrm{~S} / \mathrm{cm}$, close to the value reported for highly reduced graphene oxide [43]. Regarding rGO-graft-PTMC, the conductivity values decreased from 0.2 to $0.016 \mathrm{~S} / \mathrm{cm}$ with decreasing rGO content. It was noticed that the rGO-graft-PTMC 15 sample with $6.8 \mathrm{wt} \%$ graphene showed a similar conductivity $\left(\sim 10^{-2} \mathrm{~S} / \mathrm{cm}\right)$ as reported for PCL/rGO composites with $10 \mathrm{wt} \%$ graphene [44]. This rGO-graft-PTMC with high PTMC content could be directly used or further processed as a PTMC/rGO-graft-PTMC composite for the preparation of nerve guide conduits.

Table 3. Conductivity of rGO initiator and rGO-graft-PTMC

\begin{tabular}{|c|c|c|c|}
\hline Sample & $\begin{array}{l}\text { Residual content at } \\
500{ }^{\circ} \mathrm{C}, \%\end{array}$ & Conductivity, S/cm & Reference \\
\hline $\begin{array}{l}\text { rGO film (hydrazine- } \\
\text { reduced) }\end{array}$ & - & 2.53 & [43] \\
\hline $\begin{array}{l}\text { rGO initiator (thermally } \\
\text { reduced) }\end{array}$ & 78.2 & 1.59 & This work \\
\hline rGO-graft-PTMC 2.5 & 54.5 & 0.20 & This work \\
\hline rGO-graft-PTMC 5 & 51.6 & 0.158 & This work \\
\hline rGO-graft-PTMC 10 & 19.3 & 0.044 & This work \\
\hline rGO-graft-PTMC 15 & 6.8 & 0.016 & This work \\
\hline
\end{tabular}




\subsection{Biocompatibility}

To investigate the biocompatibility of rGO-graft-PTMC, PC12 cells were cultured on non-coated and gelatin-coated PTMC/rGO-graft-PTMC composite films. Plain PTMC films and PTMC/rGO initiator composite films were tested as well, and wells of non-tissue culture-treated culture plates were used as control. As shown in Figure 6A for non-coated surfaces, there were no differences in cell viability on all surfaces up to 3 days of culturing. On day 7, however, cell viabilities on the PTMC-based films were higher than on the culture plate. Moreover, cells cultured on PTMC/rGO-graft-PTMC films showed a significantly higher cell viability as compared to cells cultured on PTMC and PTMC/rGO initiator films. Also for gelatincoated surfaces (Figure 6B), there were only differences in cell viability on day 7. In this case, cell viabilities on the PTMC-based films were similar, and significantly lower than on the culture plate.

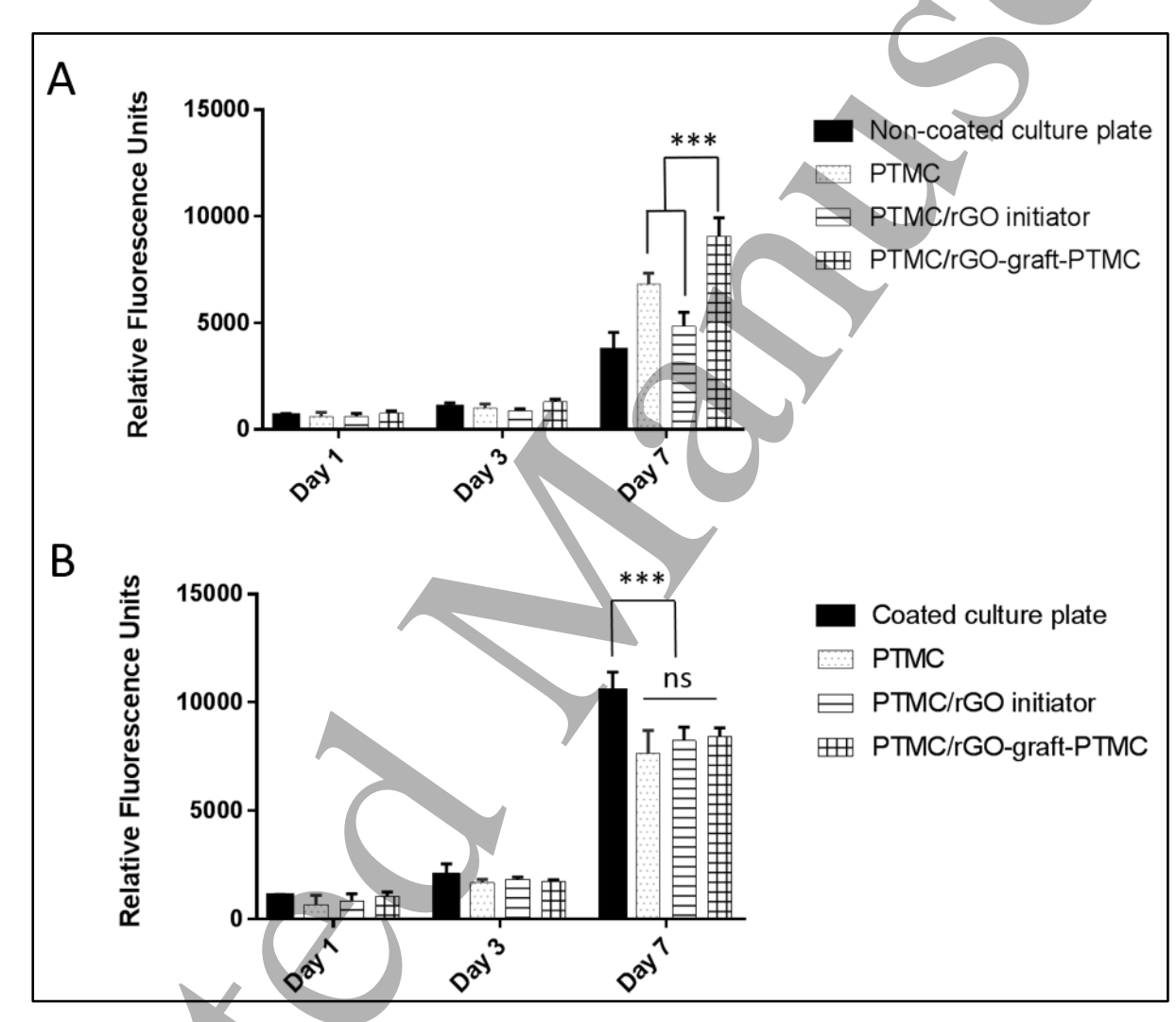

Figure 6. PC12 cell viability on non-coated (A) and gelatin-coated (B) culture plates, PTMC, PTMC/rGO initiator and PTMC/rGO-graft-PTMC composite films after 1, 3 and 7 days of culturing. The data were analyzed by two-way ANOVA. ${ }^{* * *} \mathrm{p}<0.001$, ns: no significant difference. As graphene-graft-polymer, rGOgraft-PTMC 2.5 was used.

These results were confirmed by live/dead imaging of the cells on day 7. As shown in Figure 7, a very low amount of dead cells was observed on all surfaces. In the case of non-coated surfaces, relatively low amounts of cells were observed on the culture plate and on PTMC/rGO initiator film, whereas cell numbers on PTMC and especially PTMC/rGO-graft-PTMC films were higher. All gelatin-coated surfaces showed high cell numbers, with the highest amount of cells on the culture plate. These results indicate that rGO-graft-PTMC composite is biocompatible and suitable for future application in peripheral nerve regeneration. 


\section{CONCLUSION}

rGO-graft-PTMC was successfully synthesized by ring-opening polymerization of TMC with rGO initiator. During synthesis of rGO initiator by reaction of GO with azido ethanol at high temperature, the GO was thermally reduced. Different PTMC chain lengths were obtained by using different amounts of TMC monomer in the ring-opening polymerization. During the various steps of this method, single layer graphene nanosheets remained. rGO-graft-PTMC was electrically conductive and biocompatible in culturing experiments with PC12 neuronal cells. It is concluded that rGO-graft-PTMC composite is a promising material for the preparation of nerve regeneration conduits.

\section{ACKNOWLEDGMENTS}

The authors would like to thank Huizhou Foryou Medical Company for kindly providing TMC monomer and the Chinese Scholarship Council for financial support. The authors also appreciate Jun Wang, Yang Wang and Shaochen Zhu for their help with conductivity testing and XRD measurements.

\section{REFERENCES}

[1] Wang X, So K-F and Xu X-M 2015 Advances and Challenges for Neural Regeneration Research Neural Regeneration 1 3-17

[2] Ferguson T A and Smith G M 2015 Conditions Affecting Accuracy of Peripheral Nerve Reinnervation and Functional Recovery Neural Regeneration 7 117-28

[3] Deumens R, Bozkurt A, Meek M F, Marcus M A E, Joosten E A J, Weis J and Brook G A 2010 Repairing injured peripheral nerves: Bridging the gap Prog Neurobiol 92 245-76

[4] Chiono V and Tonda-Turo C 2015 Trends in the design of nerve guidance channels in peripheral nerve tissue engineering Prog Neurobiol 131 87-104 
[5] Georgiou M, Bunting S C J, Davies H A, Loughlin A J, Golding J P and Phillips J B 2013 Engineered neural tissue for peripheral nerve repair Biomaterials 34 7335-43

[6] Gu X S, Ding F and Williams D F 2014 Neural tissue engineering options for peripheral nerve regeneration Biomaterials 35 6143-56

[7] Pateman C J, Harding A J, Glen A, Taylor C S, Christmas C R, Robinson P P, Rimmer S, Boissonade F M, Claeyssens F and Haycock J W 2015 Nerve guides manufactured from photocurable polymérs to aid peripheral nerve repair Biomaterials 49 77-89

[8] Reid A J, de Luca A C, Faroni A, Downes S, Sun M Z, Terenghi G and Kingham P J 2013 Long term peripheral nerve regeneration using a novel PCL nerve conduit Neurosci Lett 544 125-30

[9] Xie F, Li Q F, Gu B, Liu K and Shen G X 2008 In vitro and in vivo evaluation of a biodegradable chitosan-PLA composite peripheral nerve guide conduit material Microsurg 28 471-9

[10] Oh S H and Lee J H 2007 Fabrication and characterization of hydrophilized porous PLGA nerve guide conduits by a modified immersion precipitation method J Biomed Mater Res A 80a 530-8

[11] Farokhi M, Mottaghitalab F, Shokrgozar M A, Kaplan D L, Kim H W and Kundu S C 2017 Prospects of peripheral nerve tissue engineering using nerve guide conduits based on silk fibroin protein and other biopolymers Int Mater Rev 62 367-91

[12] Adamus A, Wach R A, Olejnik A K, Dzierzawska J and Rosiak J M 2012 Degradation of nerve guidance channels based on a poly(L-lactic acid) poly(trimethyléne carbonate) biomaterial Polym Degrad Stabil 97 532-40

[13] Nectow A R, Marra K G and Kaplan D L 2012 Biomaterials for the Development of Peripheral Nerve Guidance Conduits Tissue Eng Part B-Re 18 40-50

[14] Fukushima K 2016 Poly(trimethylene carbonate)-based polymers engineered for biodegradable functional biomaterials Biomater Sci-Uk 4 9-24

[15] Schuller-Ravoo S, Feijen J and Grijpma D W 2012 Flexible, elastic and tear-resistant networks prepared by photo-crosslinking poly(trimethylene carbonate) macromers Acta Biomater 83576 85

[16] Nezakati T, Cousins B G and Seifalian A M 2014 Toxicology of chemically modified graphene-based materials for medical application Arch Toxicol 88 1987-2012

[17] Tang M L, Song Q, Li N, Jiang Z Y, Huang R and Cheng G S 2013 Enhancement of electrical signaling in neural networks on graphene films Biomaterials 34 6402-11

[18] Zhu Y W, Murali S, Cai W W, Li X S, Suk J W, Potts J R and Ruoff R S 2010 Graphene and Graphene Oxide: Synthesis, Properties, and Applications Adv Mater 22 3906-24

[19] Kong Y, Zhao Y X, Ji B, Shi B B, Wei S Y, Chen G Y, Zhang L Z, Li G C and Yang Y M 2016 Preparation and Characterization of Polyacrylamide/Silk Fibroin/Graphene Oxide Composite Hydrogel for Peripheral Nerve Regeneration J Biomater Tiss Eng 6 682-9

[20] Qian Y, Zhao XT, Han Q X, Chen W, Li H and Yuan W E 2018 An integrated multi-layer 3D-fabrication of PDA/RGD coated graphene loaded PCL nanoscaffold for peripheral nerve restoration Nat Commun 9323

[21] Feng Z Q, Wang T, Zhao B, Li J C and Jin L 2015 Soft Graphene Nanofibers Designed for the Acceleration of Nerve Growth and Development Adv Mater 27 6462-8

[22] Rotman S G, Guo Z C, Grijpma D W and Poot A A 2017 Preparation and characterization of poly(trimethylene carbonate) and reduced graphene oxide composites for nerve regeneration Polym Advan Technol 28 1233-8

[23] Fang M, Wang K G, Lu H B, Yang Y L and Nutt S 2009 Covalent polymer functionalization of graphene nanosheets and mechanical properties of composites J Mater Chem 19 7098-105

[24] Min Y J, Akbulut M, Kristiansen K, Golan Y and Israelachvili J 2008 The role of interparticle and external forces in nanoparticle assembly Nat Mater 7 527-38 
[25] Wang M C, Hu N, Zhou L M and Yan C 2015 Enhanced interfacial thermal transport across graphene-polymer interfaces by grafting polymer chains Carbon 85 414-21

[26] Salavagione H J, Martinez G and Ellis G 2011 Recent Advances in the Covalent Modification of Graphene With Polymers Macromol Rapid Comm 32 1771-89

[27] Xu Z and Gao C 2010 In situ Polymerization Approach to Graphene-Reinforced Nylon-6 Composites Macromolecules 43 6716-23

[28] Layek R K and Nandi A K 2013 A review on synthesis and properties of polymer functionalized graphene Polymer 54 5087-103

[29] He H K and Gao C 2010 General Approach to Individually Dispersed, Highly Soluble, and Conductive Graphene Nanosheets Functionalized by Nitrene Chemistry Chem Mater 22 5054-64

[30] Park S and Ruoff R S 2009 Chemical methods for the production of graphenes Nat Nanotechnol 4 217-24

[31] Sumerlin B S, Tsarevsky N V, Louche G, Lee R Y and Matyjaszewski K 2005 Highly efficient "click" functionalization of poly(3-azidopropyl methacrylate) prepared by ATRPMacromolecules 38 75405

[32] Zhu K J, Hendren R W, Jensen K and Pitt C G 1991 Synthesis, Properties, and Biodegradation of Poly(1,3-Trimethylene Carbonate) Macromolecules 24 1736-40

[33] Hou Q P, Grijpma D W and Feijen J 2009 Creep-resistant elástomeric networks prepared by photocrosslinking fumaric acid monoethyl ester-functionalized poly(trimethylene carbonate) oligomers Acta Biomater 5 1543-51

[34] Gao C, He H, Zhou L, Zheng X and Zhang Y 2009 Scalable Functional Group Engineering of Carbon Nanotubes by Improved One-Step Nitrene Chemistry Chem Mater 21 360-70

[35] Pauw L J v d 1958 A method of measuring specific resistivity and Hall effect of discs of arbitrary shape Philips Research reports 13 1-9

[36] Stankovich S, Dikin D A, Piner R D, Kohlhaas K A, Kleinhammes A, Jia Y, Wu Y, Nguyen S T and Ruoff R S 2007 Synthesis of graphene-based nanosheets via chemical reduction of exfoliated graphite oxide Carbon 45 1558-65

[37] Zeng H L, Gao C and Yan D Y 2006 Poly(epsilon-caprolactone)-functionalized carbon nanotubes and their biodegradation properties Adv Funct Mater 16 812-8

[38] Gong Y N, Li D L, Fu Q and Pan C X 2015 Influence of graphene microstructures on electrochemical performance for supercapacitors Prog Nat Sci-Mater 25 379-85

[39] Mishra S K, Tripathi S N, Choudhary V and Gupta B D 2014 SPR based fibre optic ammonia gas sensor utilizing nanocomposite film of PMMA/reduced graphene oxide prepared by in situ polymerization Sensor Actuat B-Chem 199 190-200

[40] Fang M, Wang K G, Lu H B, Yang Y L and Nutt S 2010 Single-layer graphene nanosheets with controlled grafting of polymer chains J Mater Chem 20 1982-92

[41] Song M Y, Yun Y S, Kim N R and Jin H J 2016 Dispersion stability of chemically reduced graphene oxide nanoribbons in organic solvents Rsc Adv 6 19389-93

[42] Tang L C, Wan Y J, Yan D, Pei Y B, Zhao L, Li Y B, Wu L B, Jiang J X and Lai G Q 2013 The effect of graphene dispersion on the mechanical properties of graphene/epoxy composites Carbon 6016 27

[43] Park S, Suk J W, An J H, Oh J, Lee S, Lee W, Potts J R, Byun J H and Ruoff R S 2012 The effect of concentration of graphene nanoplatelets on mechanical and electrical properties of reduced graphene oxide papers Carbon 50 4573-8

[44] Sayyar S, Murray E, Thompson B C, Gambhir S, Officer D L and Wallace G G 2013 Covalently linked biocompatible graphene/polycaprolactone composites for tissue engineering Carbon 52 296-304 